\title{
The Mini-Cog compares well with longer screening tests for detecting dementia in older people
}

Borson S, Scanlan JM, Chen P, Ganguli M. The Mini-Cog as a screen for dementia: validation in a population-based sample.

J Am Geriatr Soc 2003;51:1451-4.

\section{Does the Mini-Cog, a rapid cognitive screening test, accurately detect dementia in older people?}

\section{METHODS}

Design: Prospective cohort study.

(2)

Setting: 23 rural communities, Pennsylvania, USA; recruitment initiated early 1990s.

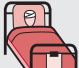

Patients: A subset of 1119 elderly people (mean age 73 years) randomly drawn from an age stratified random sample of 17000 people from the Monongahela Valley Independent Elders Survey (MoVIES). Participants were aged $>65$ years, had a minimum of 6 years of formal education, and were living in the community.

Test: The Mini-Cog is a rapid screening test comprising two cognitive tasks: a three item word memory test and clock drawing. The scores were combined with an empirical algorithm to yield a dementia screen score for each person as either "possibly impaired" or "probably normal".

Diagnostic standard: The Mini-Mental State Examination (MMSE)

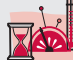

Outcomes: Sensitivity and specificity of the Mini-Cog.

\section{MAIN RESULTS}

Seventy six people (6.4\%) met criteria for dementia (DSM-III-R). The Mini-Cog had higher sensitivity but lower specificity than the MMSE

Table Sensitivity, specificity, and assessment time of dementia screening tests

\begin{tabular}{llll}
\hline Diagnostic test & $\begin{array}{l}\text { Sensitivity } \\
(\%)\end{array}$ & $\begin{array}{l}\text { Specificity } \\
(\%)\end{array}$ & $\begin{array}{c}\text { Assessment time } \\
\text { (minutes) }\end{array}$ \\
\hline $\begin{array}{l}\text { MoVIES neurocognitive } \\
\text { battery }\end{array}$ & 75 & 90 & $\geqslant 30$ \\
Mini-Cog & 76 & 89 & $2-4$ \\
MMSE & & & $5-12$ \\
$23 / 24$ cut off & 71 & 94 & \\
$24 / 25$ cut off & 79 & 88 & \\
\hline
\end{tabular}

For correspondence: Dr S Borson, University of Washington School of Medicine, Seattle, WA 98195, USA; soob@u.washington.edu

Sources of funding: supported by the National Institute on Aging. using the generally applied MMSE cut off of 24. Specificity and sensitivity were similar when the MMSE cut off was raised to 25 (see table). The Mini-Cog had a shorter administration time.

\section{CONCLUSIONS}

Sensitivity and specificity of the Mini-Cog compared well with that of the MMSE, and longer screening tests.

\section{Commentary}

$\Delta$ $s$ more treatments for dementia become available, screening for dementia in general medical practice becomes a mandate. A good screening test should be sensitive, specific, brief, easy to administer, and free of confounding demographic variables.' The authors of the Mini-Cog have chosen three word recall and clock drawing from a population based post hoc examination of the data as a screen for dementia, comparable in classification with the MMSE. Although the sensitivity for each measure alone was modest, combining the tests improved sensitivity by 17-22 points, with a 6 point reduction in specificity. If, going forward, this screen is administered with the clock drawing task interposed between the introduction of the words and their recall, this screen would meet many of the necessary proposed criteria.

Although word recall addresses the memory deficits that are often the first symptom of Alzheimer's disease, adding the clock drawing task makes possible the identification of individuals with salient visuomotor or executive dysfunction and less obvious memory deficits, possibly due to cerebrovascular or frontotemporal dementing disorders. However, the optimal cut off score for the Mini-Cog results in a positive predictive value of $32 \%$, indicating that only one of every three identified people is actually impaired (at their base rate of $6.4 \%$ ). When we compared the three word recall with the 4 item Memory Impairment Screen (MIS), we found that, by using a controlled learning procedure, which required the examinee to match each word to be remembered with its category cue followed by using both free and cued recall at retrieval, ${ }^{2}$ sensitivity was 0.86 and specificity 0.97 , yielding a PPV of 0.66 at $6.4 \%$ base rate. ${ }^{3}$ The MIS alone did not differ significantly from the MMSE in sensitivity and specificity. ${ }^{4}$ Adding controlled learning with free and cued recall at retrieval would improve the three item recall portion of the Mini-Cog reducing the number of individuals $(68 \%)$ positively identified in error which, at present, may reduce relevance to practice.

Gail Kuslansky, PhD Albert Einstein College of Medicine, Bronx, NY 10461, USA

1 Gifford DR, Cummings JL. Evaluating dementia screening tests. Neurol 1999;52:224-7.

2 Buschke $H$, Kuslansky $G$, Katz M, ef al. Screening for dementia with the memory impairment screen (MIS). Neurol 1999:52:231-7.

3 Kuslansky G, Buschke H, Katz M, et al. Screening for Alzheimer's disease: the Memory Impairment Screen (MIS) versus the conventional three-word memory test. J Am Geriat Soc 2000;50:1086-91.

4 Kuslansky G, Buschke $H$, Katz M, et al. Community based screening for dementia with the Memory Impairment Screen and the Mini-Mental State Examination. Neurol 2000;58:A104. 